\title{
Perception of voicing and place features in whispered speech: A dichotic choice analysis
}

\author{
JOHN ALLEN \\ Laboratory of Experimental Psychology, University of Sussex, Brighton BN1 9QY, England \\ and \\ MARK HAGGARD \\ Department of Psychology, Queen's University, Belfast BT7 INN, Northern Ireland
}

\begin{abstract}
To offset shortcomings of existing demonstrations of right-ear superiority in the analysis of formant transitions, an experiment was performed on whispered speech. Two aspects of dichotic listening performance were examined in a single-report paradigm: the right-ear advantage (REA) for the perception of the voicing distinction and the feature sharing advantage (FSA) for both voicing and place features. A significant REA was obtained for the voicing distinction cued by first formant transition in the absence of a switch from aperiodic to periodic excitation. This, plus a greater incidence of voiced responses to right-ear stimuli, suggests that a distinction involving transitions can specifically augment the REA. The data also showed better identification of place and of voicing feature values when the competing dichotic speech stimuli shared these respective features (FSA) than when they did not. This FSA was restricted to the feature shared and hence not an effect of response uncertainty. The implications of these results for models of speech processing are discussed.
\end{abstract}

Dichotic studies controlling the acoustic-phonetic properties of speech have had two major objectives: to determine the factors in the stimulus and task that predispose the right-ear advantage (REA) and to assess the nature of the binaural interaction in terms of fusion, contralateral masking, and other forms of competition, which leads to the low percentage of correct reports for synchronized dichotic syllables. The present experiment was designed primarily to assess stimulus determinants of the REA. However, by employing a more refined analysis than is generally used in dichotic experiments, it also provides some insight into the nature of dichotic interference.

Haggard and Parkinson (1971) reported an experiment in which a substantial REA was obtained for the voicing feature distinction with a set of stimuli whose acoustical cues to that distinction were limited to variations in a single secondary cue, the trajectory of the fundamental frequency (Haggard, Ambler, \& Callow, 1970). In accounting for REA at that time, some emphasis was laid (Haggard, 1971; Liberman, Cooper, Shankweiler, \& StuddertKennedy, 1967) upon the property of "encoding" (lack of contextual invariance) in such important speech cues as the major cue to the voicing

The authors thank St. Dunstan's and the Joint Speech Research Unit for support. J. Allen is now at Central School of Speech and Drama, Eton Avenue, London NW3 3HY. M. Haggard is now at MRC Institute of Hearing Research, University of Nottingham, England, to where reprint requests should be sent. distinction, namely voice onset time (VOT) (Lisker \& Abramson, 1964). The fundamental frequency trajectory shows a relative degree of invariance in speech; while the absolute value of VOT covaries with the place of an initial voiced stop, particularly within the voiceless set $/ \mathrm{p}, \mathrm{t}, \mathrm{k} /$, such covariation is not observed in the absolute or relative values of fundamental frequency of the onset of voicing (Haycock \& Haggard, Note 1). In the light of these data, Haggard reasoned that the role of acoustical cues in carrying a linguistic distinction with the experiment itself was within limits unimportant. Instead, it was argued that possibly the mere presence of certain acoustical properties of a syllable, associated with one value of a normally encoded feature, might trigger activity of a left-hemisphere phonetic processor, even though the experimental task might not require the use of variations in the property as a cue to a phonemic distinction. Any acoustical property, it was suggested, might activate lefthemisphere mechanisms in this way, providing that it might serve as an encoded cue to phonemic contrast in a natural speech context, i.e., in a situation where the number of phonetic distinctions was not artificially restricted. For voicing, which normally gives a slightly greater REA than place of articulation (Studdert-Kennedy \& Shankweiler, 1970; Studdert-Kennedy, Shankweiler, \& Pisoni, 1972) such a property might be the onset of periodic voice excitation. This is defined as the switch from a voiceless mode of aperiodic vocal tract excitation, as in 
burst or aspiration, to one with predominantly low-frequency harmonic excitation from the vocal cords. Values of delay (VOT) at this onset after the initial plosive burst of greater than about $20 \mathrm{msec}$ typically give perception of a voiceless phoneme, shorter values giving a voiced percept. The possibility of a triggering role for VOT can be tested by using whispered speech. There can be no such abrupt switch of excitation in whispered speech, which has aperiodic excitation throughout. Nevertheless, the voicing distinction survives on secondary cues related to the relative predominance of low- and high-frequency portions of the spectrum. In particular, the greater intensity of the first formant region, and hence the greater detectability of the first formant transition for voiced phonemes (Liberman, Delattre, \& Cooper, 1958; Stevens \& Klatt, 1974; Summerfield \& Haggard, 1974), should be almost as valid a cue as in normal speech.

The voicing distinction in whispered speech of fers a powerful possibility for testing the specificity of the REA to certain analyzers of acoustical properties characteristic of speech. Firstly, if no REA were obtained with whispered speech, the role of voicing onset as a triggering feature would be strongly implicated (Haggard \& Parkinson, 1971). Secondly, if a marked REA were obtained, then left-hemisphere detection of the first formant transition would be strongly implicated. Such involvement could be shown in either or both of two ways. First, greater right-ear discrimination of presence and absence of the transition could lead to overall higher manifest accuracy of report for the feature. Second, greater right-ear ability to detect transitions when present (a threshold or biasing effect) could yield more instances of the voiced value in responses to right-ear stimuli. Without this distinction, it would be impossible, for example, to rule out as an explanation of REA some more optimal proportion of voiced responses. Dichotic experiments have been generally lax in such precautions, which, though unnecessary for qualitative demonstrations, are a prerequisite for the sort of detailed interpretation attempted here.

There is some need for a specific test for an REA (left-hemisphere dominance) for properties such as transitions. Cutting (1974), in an ingenious variation of the speechlikeness of stimuli with a consonant-vowel (CV) format, showed that inclusion of transitions and global speechlikeness both appeared to influence the magnitude of the REA. In his experiment, REA was measured, not by identification consistency, but by subjective preposition of the right-ear stimulus in a temporal order judgment. While the two measures of REA in general correlate well, it is possible that the processing time for transitions and steady states interacts differently with the storage capacities of the two ear-hemisphere systems (cf. Allen \& Haggard, 1977) and that the asymmetry, though related to asymmetrical processing for speech, is not related to transition properties as such. Another limitation to the interpretation of Cutting's result is the fact that a task context predisposing a linguistic set may override stimulus factors as determinants of REA when these are not fully natural or leave room for both linguistic and nonlinguistic modes (Kinsbourne, 1973; Spellacy \& Blumstein, 1970). Trial-to-trial variations in some apparently important stimulus properties do not lead to trial-to-trial variations in the REA (Darwin, 1971; Haggard, 1971). As Cutting's conditions were blocked, it is possible that in a cumulative fashion over the experiment the presence of transitions in the less speechlike stimuli (frequency-modulated sine-wave complexes) could have constituted simply an additional, statistical, inducement to subjects to code the stimuli in speech mode, because of their globally greater similarity to speech patterns, rather than because of specific events in processing the actual occurrences of the stimuli (cf. Haggard, 1977). Not only is it important to distinguish between factors of linguistic set or strategy and factors specific to stimulus processing, but an experiment by Ades (1974) renders desirable some independent corroboration of asymmetry for the analysis of transitions. Ades adapted the two ear-hemisphere systems differentially, with different dichotic CV stimuli showing that there was bilaterality of at least the analysis of such properties as transitions. Stronger evidence is consequently required to sustain a belief in the asymmetry of their phonetic mapping.

The foregoing discussion of asymmetrical processing has implicitly assumed that the aspect of perception relevant to REA is the processing of the binary or trinary features, not the occurrence of stimulus patterns for the particular phonemes that are whole bundles of feature values. An explicit validation of that assumption is lacking, but the evidence that values of different features from the two ears combine with significantly greater than chance frequency in dichotic experiments (StuddertKennedy \& Shankweiler, 1970) is powerful evidence for a model involving relatively great independence of feature processing at some stage. It appears that the two presented values of the acoustical cues to each feature are in some sense summated. If they differ little, they fuse psychoacoustically (Cutting, 1976) and the addition is of a rather direct sort. If the difference is great, there is statistical competition at a later stage (Haggard, 1977), with advantage for the right ear stimulus and, in staggered presentation, for the later stimulus. Accuracy of report is greater when feature values are shared on the two ears (Studdert-Kennedy et al., 1972). This fact is obvious whether one deduces it directly from a 
summation (integration) model of contralateral masking, as above, or seeks to explain it in terms of fewer combinations of feature values to map onto a pair of responses in the task, and it is variations in the effect that are chiefly of interest. The explanation in terms of mapping uncertainty may not be necessary, in that Pisoni and McNabb (1974) have obtained this "feature-sharing advantage" (FSA) for the voicing feature with the more powerful masking paradigm involving attention to one ear and single report. They showed that the FSA is restricted to the particular feature shared. This finding points towards the first explanation, in terms of perceptual integration, and also towards an independence of the accumulators of feature evidence in this integration process; the conflict which leads to poorer dichotic than diotic performance remains on one feature even when there is no conflict for the other and it is displaying FSA.

It is worthwhile to demonstrate the restriction of FSA to the feature shared for further features such as place, which was never shared by target and mask stimuli in Pisoni and McNabb's experiment. But such a demonstration has particular value here in the context of the foregoing argument about the transition cue to voicing. Detectability of all formant transitions, not merely the first, appears to have positive cue value for voicing (Summerfield \& Haggard, 1974), yet the direction of second and third formant transitions is primarily associated with the place distinction and presumed to be related to the basis of its REA, irrespective of voicing. To show that REA is obtained for processing of the voicing distinction and not strictly for the stimuli as global entities, it is desirable to show that independent feature processing as suggested by FSA is operating for both features. Accordingly, an experiment was designed on the voicing feature in whispered speech with stimuli also varying on the place dimension. A single-report paradigm was employed, since it permits analysis in terms of the separate features by pooling of the confusion matrices. To separate the influences contributing to the percentage correct score, we had to analyze these matrices in a fashion that separated discriminability from bias factors. We were particularly interested in whether the REA would occur in both discriminability and bias parameters (or neither, or both), given its special acoustical basis in whispered speech, and whether FSA would be shown for the place feature.

\section{METHOD}

\section{Stimuli}

The four CV syllables / ba, da, pa, ta/ were generated on the digital speech synthesizer at Sussex University. Aperiodic excitation was therefore used throughout each of the four utterances. The acoustical parameters employed for synthesizing the four syllables were adjusted so as to be most appropriate for this whispered speech. Schematic spectrograms of the stimuli are given in Figure 1.

The voiceless stop consonants were synthesized with the onset of the first formant (F1) delayed relative to the onset of the second and third formants. This $F 1$ cutback results in a reduction in the extent (and hence detectability) of the first-formant transition and is an effective cue to the voicelessness (Stevens \& Klatt, 1974). To enhance the naturalness of the two voiced stop consonants, it was also found necessary to reduce the amplitude of the initial noise burst below that generally used in the synthesis of voiceless stop consonant stimuli.

The total duration of each CV syllable was $220 \mathrm{msec}$. In each syllable, a 20 -msec silent segment separated an initial noise burst from a 80 -msec formant transition to the following $(110-\mathrm{msec})$ steady-state vowel. In the phonemically voiceless stop consonantvowel syllables, the first formant commenced at the termination of the second-formant transition.

A dichotic tape was generated by recording all the possible pairings of the four syllables (no syllable was paired with itself) 10 times in a random sequence of 60 dichotic trials. A second 60-trial sequence was recorded by reversing the order of the stimuli. The intertrial interval was $6 \mathrm{sec}$.

\section{Subjects}

Twelve right-handed undergraduate volunteers acted as subjects. None had any known hearing loss and all were native speakers of English. They were paid for participating.

\section{Procedure}

As a preliminary test of level of performance, each subject heard a 60-trial binaural recorded sequence. The stimuli were presented binaurally over headphones (Sharp HA-1011). The subjects recorded their identifications by checking a response sheet in one of four columns headed by the labels "pa, ta, ba, da." No feedback of results was given.

Following the binaural identification trials, the dichotic test was administered. The subjects were informed that they would receive two of the four syllables on each trial, one on the left ear and one on the right ear. They were also told that the two
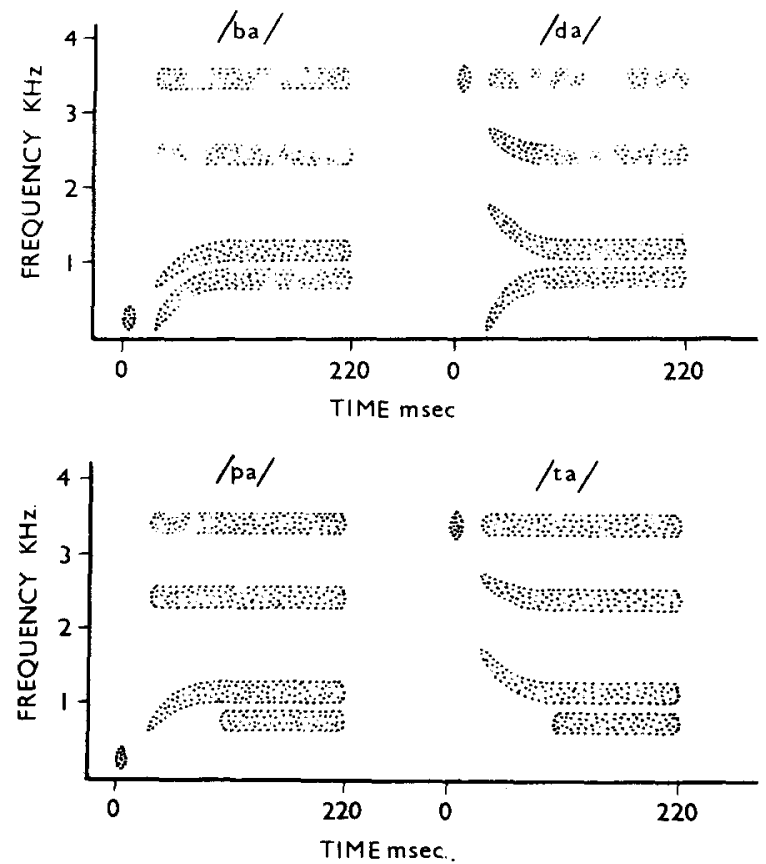

Figure 1. Schematic spectrograms of the stimuli /ba, da, pa, ta/. 
syllables on each trial would arrive simultaneously. All subjects were played the dichotic tape twice, giving four blocks of 60 trials each. At the beginning of each block of trials, they were instructed to attend to the ear designated and report only the syllable they heard on that ear. Responses were written on prepared response sheets. Half the subjects received instructions to attend over the four blocks according to the order RLLR, while the remainder followed the order LRRL. Track/earphone to ear assignment was reversed within and across subjects at half time. Brief rest pauses were taken between each block of trials. Twenty dichotic practice trials were given prior to the dichotic test proper.

The two channels of the tape recorder were equated for gain, prior to each experimental session, by monitoring the output while playing the recording of a computer-generated calibration tone:

\section{RESULTS}

\section{Binaural Identification Test}

The mean percentage of correct responses on the binaural identification test was $80 \%$. No subject scored less than $75 \%$ correct. In order to determine the effectiveness with which the stimuli signaled the voicing and place features, the identification responses were scored for each feature separately, pooling over values of the other features. The mean accuracies with which the subjects correctly assigned a voiced response and voiceless responses were $92 \%$ and $90.3 \%$. The mean accuracies for the bilabial and alucolar values of the place feature were $86.6 \%$ and $95.5 \%$. A Wilcoxon $\mathrm{T}$ test on the numbers of voiced and voiceless responses revealed that there was no significant bias towards either (voiced or voiceless) response. Hence, the stimulus parameters had been suitably adjusted to span the category boundary for the voicing distinction.

\section{Dichotic Test}

The responses from the dichotic test were analyzed by means of a computer program especially written for this purpose.

For each of the 12 subjects, the program produced a 4 by 4 confusion matrix for each ear based upon 120 trials. From each primary matrix, two pairwise submatrices were extracted for the phonemes contrasting in voicing alone (the $/ \mathrm{p} /-/ \mathrm{b} /$ and $/ \mathrm{t} /-/ \mathrm{d} /$ discriminations) and two for those differing in place alone $(/ \mathrm{p} /-/ \mathrm{t} /$ and $/ \mathrm{b} /-/ \mathrm{d} /$ discriminations $)$. Although this uses less data for computation of each performance measure than simply collapsing the irrelevant feature, it preserves a check on the validity of such collapsing after computation. The entries for the submatrices were obtained by tabulating the correct response frequencies for each stimulus for the data obtained over all the trials. The measures of discriminability and bias computed from these were $\log _{e} \alpha$ and $\log _{\mathrm{e}} \mathrm{v}$ derived from Luce (1959). $\log _{\mathrm{e}} \alpha$ and $\log _{\mathrm{e}} \mathrm{v}$ are similar to the $\mathrm{d}^{\prime}$ and $\log \beta$ parameters, respectively, of signal detection theory. A general introduction to the derivation and significance of these parameters is given by Broadbent
(1971), and their use in studies of speech perception has been discussed by Haggard (1968).

Before examining the dichotic discriminability and bias estimates, however, it is worth noting that the mean percentage of wholly correct responses (i.e., correct for both voicing and place) were 36.6 and 49.6 under the left- and right-ear conditions, respectively. This low overall level of performance reflects the intrinsic difficulty of the dichotic task. The $13 \%$ right-ear advantage on overall percent correct was significant (Wilcoxon $T=7, n=11$, $p<.01)$. All responses were also scored for the correct values of the voicing and place features separately. The particular value of the feature in the response not under consideration was ignored in this preliminary analysis. For left and right ears, the mean percentage of all trials on which the voicing feature was correctly reported were $63.1 \%$ and $68.1 \%$, respectively. The corresponding place figures were $59.4 \%(\mathrm{~L})$ and $69.6 \%(\mathrm{R})$. The right-ear advantage for voicing was significant $(T=17, n=12$, $\mathrm{p}<.05$; one-tailed). However, although the ear asymmetry for the place feature was larger than that for the voicing feature, the greater variability in the place scores prevented the ear advantage for this feature from reaching a conventional level of significance.

The means of the discriminability estimates $\left(\log _{\mathrm{e}} \alpha\right)$ for the stimuli contrasting in voicing alone are presented in Table 1.

The $\log _{\mathrm{e}} \alpha$ values are all positive, showing that subjects could adequately use the F1 cutback and burst amplitude cues to voicing, even during dichotic presentation. Wilcoxon $T$ tests confirmed that the right-ear voicing discriminability scores were significantly superior to those for the left ear for both the $/ \mathrm{p} /-/ \mathrm{b} /$ and $/ \mathrm{t} /-/ \mathrm{d} /$ contrasts $(\mathrm{T}=5, \mathrm{n}=12$, $\mathrm{p}<.01 ; \mathrm{T}=12, \mathrm{n}=12, \mathrm{p}<.05$ ). The extent of this right-ear superiority was significantly greater for the $/ \mathrm{p} /-/ \mathrm{b} /$ than for the $/ \mathrm{t} /-/ \mathrm{d} /$ contrasts $(\mathrm{T}=10.5, \mathrm{n}=12, \mathrm{p}<.05)$.

The data on voicing biases are presented in Table 2 , in which a value of $\log _{\mathrm{e}} \mathrm{v}$ greater than zero reflects a bias towards a voiced response. Looking at the

Table 1

Voicing Discriminability: Means of Individual $\log _{e} a$ Values

\begin{tabular}{lccc}
\hline & Left Ear & Right Ear & $\mathbf{R}-\mathbf{L}$ \\
\hline$/ \mathrm{p} /-\mathrm{b} /$ & 1.31 & 2.02 & .71 \\
$/ \mathrm{t} /-\mathrm{d} /$ & 1.34 & 1.66 & .32 \\
\hline
\end{tabular}

Table 2

Voicing Bias: Means of Individual $\log _{e} v$ Values

\begin{tabular}{lccc}
\hline & Left Ear & Right Ear & R - L \\
\hline$/ \mathrm{p} /-\mathrm{lb} /$ & .35 & .77 & .42 \\
$\mid \mathrm{t} / / \mathrm{d} /$ & .55 & .77 & .22 \\
\hline
\end{tabular}

Note-The positive values of the $\log _{e} v$ indicate a slight bias toward voiced responses, overall. 
right-ear data, it is obvious that the greater right-ear advantage for voicing in the $/ \mathrm{p} /-/ \mathrm{b} /$ stimuli cannot be explained by more optimal (i.e., on-phonemeboundary) conditions as the right ear bias value, 0.77 , is the same for the two places of articulation and the left-ear values differ only slightly. The tendency to produce more voiced responses when attending to the right as opposed to the left ear reached significance only for the $/ p /$ and $/ b /$ stimuli $(\mathrm{T}=0, \mathrm{n}=12, \mathrm{p}<.01)$. For the $/ \mathrm{t} / \mathrm{and}$ /d/ stimuli, the bias towards voiced responses was not significantly greater when subjects attended to the right ear.

Table 3 presents the discriminability values for the place distinction. Due to high subject variability arising from few entries in some cells, the right-ear advantage for place of articulation reached significance only for the voiceless stimuli $/ p /$ and $/ \mathrm{t} / \quad(\mathrm{T}=15, \quad \mathrm{n}=12, \quad \mathrm{p}<.05 ; \quad$ one-tailed, Wilcoxon).

The bias estimates for place of articulation are given in Table 4. There was no significant difference between the ears for either the $/ b /-/ d /$ or $/ \mathrm{p} /-/ \mathrm{t} / \log _{\mathrm{e}} \mathrm{v}$ values.

The effect of feature sharing upon identification accuracy was examined by analyzing the responses separately for trials on which the dichotic stimuli (a) shared the same voicing feature, (b) shared the same place feature, and (c) shared neither place nor voicing features. The results of this analysis (for responses which were correct for voicing and place) are presented in Table 5. Identification accuracy (calculated using the responses pooled over both conditions of attention) was significantly better on place-shared trials than on trials which shared neither voicing nor place features $(\mathrm{T}=0, \mathrm{n}=12, \mathrm{p}<.01)$. Similarly, identification accuracy on voicing-shared pairs was significantly superior to that on trials sharing neither feature $(T=13, n=12, p<.05)$. Response accuracy was also significantly better on place-shared pairs than on voicing-shared pairs ( $\mathrm{T}=7.5, \mathrm{n}=12, \mathrm{p}<.02$ ). Thus, as in StuddertKennedy and Shankweiler's (1970) study, greater FSA accrued to pairs sharing place than to pairs

Table 3

Place Discriminability: Means of Individual $\log _{\mathrm{e}} \alpha$ Values

\begin{tabular}{lccc}
\hline & Left Ear & Right Ear & $\mathrm{R}-\mathrm{L}$ \\
\hline$/ \mathrm{b} /-/ \mathrm{d} /$ & 1.33 & 1.78 & .45 \\
$/ \mathrm{p} /-\mathrm{t} /$ & 1.14 & 1.74 & .60 \\
\hline
\end{tabular}

Table 4

Place Bias: Means of Individual $\log _{\mathbf{e}} \mathrm{V}$ Values

\begin{tabular}{lccc}
\hline & Left Ear & Right Ear & R $-\mathrm{L}$ \\
\hline$/ \mathrm{b} /-/ \mathrm{d} /$ & .55 & .65 & -.1 \\
$/ \mathrm{p} /-/ \mathrm{t} /$ & 1.44 & 1.00 & .44 \\
\hline
\end{tabular}

Note-Positive values indicate a predominance of bilabial $(/ p, b /)$ responses.
Table 5

Mean Percentages of Correct Responses on Trials Sharing Place, Voicing, or Neither Feature

\begin{tabular}{lccc}
\hline Ear & Place & Voicing & $\begin{array}{c}\text { Neither } \\
\text { Feature }\end{array}$ \\
\hline R & 59.9 & 44.6 & 39.8 \\
L & 45.9 & 36.5 & 27.7 \\
R + L & 52.9 & 40.5 & 33.7 \\
R - L & 14.0 & 8.1 & 12.1 \\
\hline
\end{tabular}

Table 6

Feature Analysis: Mean Percentages Correct on Trials Sharing Place, Voicing, or Neither Feature

\begin{tabular}{lccc}
\hline & Place & Voicing & $\begin{array}{l}\text { Neither } \\
\text { Feature }\end{array}$ \\
\hline $\begin{array}{l}\text { Place pooled } \\
\text { Voicing correct }\end{array}$ & 54.9 & 87.0 & 54.0 \\
$\begin{array}{l}\text { Voicing pooled } \\
\text { Place correct }\end{array}$ & 90.9 & 51.1 & 53.4 \\
\hline
\end{tabular}

sharing voicing. Wilcoxon $\mathrm{T}$ tests on the left- and right-ear scores obtained under the place-shared, voicing-shared, and nonshared conditions revealed that the right-ear advantage reached significance only on pairs sharing place or neither feature $(\mathrm{T}=11, \mathrm{n}=11, \mathrm{p}<.05$, and $\mathrm{T}=9, \mathrm{n}=11$, $\mathrm{p}<.05$, respectively). This is also consistent with Studdert-Kennedy et al.'s (1972) result.

Finally, the responses were scored for voicing and place correct separately on all three types of dichotic trials. In this analysis, the feature not under consideration was made irrelevant for the purpose of scoring responses. The results of this analysis (pooled over both ear conditions) are presented in Table 6. All subjects obtained place-correct scores on placeshared pairs which were significantly superior to those achieved on pairs which shared voicing $(p<.001$; sign test) or neither feature $(p<.001)$. Indeed, as Table 6 shows, on place-shared pairs the place feature was correctly incorporated into the subjects' responses on over $90 \%$ of all such trials. Similarly, without exception, voicing correct scores were significantly better on pairs sharing this feature than on pairs sharing place or neither feature $(p<.001$ in each case). The symmetry of these results suggests very strongly that the feature-sharing advantage is restricted to the feature shared.

\section{DISCUSSION}

The results of this experiment give no support for the hypothesis that a switch in excitation mode is necessary to reveal a right-ear advantage for the perception of voicing. A reliable right-ear superiority for the voicing distinction can be demonstrated in the absence of a change from aspiration, or silence, to periodic vibration. This result clearly does not favor the triggering hypothesis, since the right-ear 
advantage for voicing is not tied to that acoustical characteristic thought most likely to qualify as a trigger feature-namely, the onset of periodic excitation. Likewise, the result gives little support to the idea that encodedness of the stimulus properties is important, because, while extent of F1 transition varies with vowel context, it does not vary with the place of articulation.

The REA for voicing when the major voicing cue is $F 1$ cutback suggests that this cue can override the absence of the cues normally associated with the onset of voicing. This is interesting theoretically, since it indicates that the higher performance on voicing decisions by left-hemisphere speech mechanisms does not require the summation of evidence from all the natural voicing cues. It appears that if a feature such as F1 cutback alone may suffice to cue the voicing distinction, it is also sufficient to reveal a right-ear advantage. It is possible that the magnitude of the REA for voicing cued by F1 cutback alone is less than that obtained with stimuli which incorporate F1 cutback and voice onset delay. Studdert-Kennedy and Shankweiler (1970) found, for example, that natural stop consonant stimuli yielded a $12 \%$ REA for this feature as compared with the $5 \%$ REA obtained in the present experiment. However, the high intersubject variation normally encountered in dichotic studies of ear differences prevents us drawing firm conclusions from such comparisons. The similarity of the REA effects as a function of particular feature shared to those obtained by Studdert-Kennedy et al. (1972) is remarkable given that their vowel contexts, $/ \mathrm{i} /$ and $/ \mathrm{u} /$, had no $F 1$ transition and hence the voicing distinction relied exclusively on VOT. Despite the aforementioned consistency, the data from the discriminability analysis does not give us the sought evidence for left-hemisphere specialization in processing transitions. Lisker (1974) and Summerfield (1975) have recently shown that the $F 1$ cutback cue is primarily one of absolute onset frequency, not transition rate, duration, or extent. Summerfield further argues that the relative timing of onsets in high- and low-frequency regions, whether transitional or not, could be a global descriptor encompassing several of the known acoustical cues to voicing. Such formulations do not themselves rule out a role for transitions, but they provoke the counterdemonstration of a perceptual asymmetry not ascribable to better left-hemisphere discriminability of relative onset time of $F 1$ and higher formants. The significantly greater number of voiced responses made while subjects attended to the right ear constitutes such a demonstration. This result suggests that the right-ear/left-hemisphere system is more responsive to the presence of $F 1$ in the early portion of $\mathrm{CV}$ syllable than is the left-ear/righthemisphere system. This gives direct support to the idea that the right-ear/left-hemisphere system displays some degree of specialization for processing transition information, as suggested by Myers (1970).

The results of the feature sharing analysis replicate and extend the findings reported by Pisoni and $\mathrm{McNabb}$ (1974). It is clear that a substantial FSA arises even when subjects are required to make a single response on each dichotic trial. According to Studdert-Kennedy et al. (1972), the feature-sharing advantage arises because "activation of a feature processor for one response facilitates its activation for another temporally contiguous response." Thus, the effect is held by these authors to arise at a stage immediately prior to the output of two separate phonemic responses. However, the present findings strongly support Pisoni's observation that the effect does not arise through activation of a unit controlling a response, since the advantage persists even when a second response is not required. The implication, then, is that the feature-sharing advantage arises at a stage when either acoustic or phonetic features are being extracted or stored. The distinction between these different stages is made explicit in Figure 2, in which a schematic representation of some of the stages involved in the process of phonetic recognition is presented. According to this scheme, auditory features are extracted and stored prior to phonetic feature analysis. The insertion of an auditory buffer store after the stage of preliminary auditory analysis is consistent with a large body of evidence concerning the role of auditory memory in speech perception (Allen, 1976; Darwin \& Baddeley, 1974; Massaro, 1972).

The inclusion of a feature buffer store in Figure 2 follows a suggestion by Pisoni (1975), who argues that the feature-sharing advantage arises after phonetic feature analysis and while extracted phonetic features are held in a short-term register. Pisoni invoked the notion of a phonetic feature buffer store for two principal reasons. First, he assumed that not all phonetic features may be processed at the same rate. Secondly, he suggested that features have to be preserved independently for subsequent stages of phonological analysis. However, replicated or redundant features would not need to be maintained separately in this short-term store, so confusions between inputs sharing a phonetic feature would be less likely to occur.

The suggestion that FSA arises during phonetic extraction or storage is weakly consistent with the

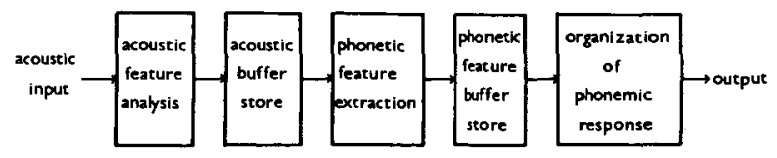

Figure 2. Schematic representation of some of the stages involved in the process of phonetic recognition. 
fact that FSA is restricted to the particular feature shared. But the phonetic interpretation is based chiefly on the contention that FSA is unaffected by contextually conditional acoustical factors, and one aspect of the present data threatens this. The gain which accrued on trials sharing voicing was significantly less than that on trials sharing place of articulation despite similar overall binaural intelligibility levels. This outcome is difficult to account for on a purely phonetic basis, as there would then be no reason why voicing-shared pairs would give rise to a iesser advantage than place-shared trials. It is possible to argue similarly from other data without using the FSA concept. With poor information as to ear, the response in a dichotic masking situation involves a form of summation of the feature values, one of which precipitates "errors" in the response. The level of performance in backward and simultaneous dichotic masking is in general substantially influenced by acoustical similarity (Porter \& Mirabile, in press). Because the relation between spectrum and phoneme is not entirely abstract, it may be difficult to design a structured set of stimuli in which acoustic bases of a phonemic distinction are so different that further acoustic variations could be made in them with no effect upon masking performance while still allowing phonetic summation to play a role.

In explaining the discrepant FSA for the two features, we are inclined to invoke a dimension of competition/fusion based upon spectral similarity (Haggard, 1977). Pisoni (1975) noted less interference from contralateral features when the vowel context and hence the spectral similarity of the syllables on the two ears differed, but had no satisfactory explanation of it. Studdert-Kennedy et al. (1972) based the phonetic level pooling of features upon similar FSA for $/ i /$ and $/ u /$, but these are acoustically dissimilar only in the second-formant trajectory, sharing a first-formant trajectory. Furthermore, both in natural speech and in their stimuli, the rising third-formant trajectory in /bu/ overlaps the rising second-formant trajectory in $/ \mathrm{bi} /$, possibly explaining the lack of dependence of FSA upon vowel context. It is perhaps more appropriate to treat FSA as a fortuitously fortunate special case of the general process of intrusions of feature values from the unattended ear. While place and voicing remain to a first-order approximation independent, in dichotic intrusions, Paul and Haggard (1975) have shown that consonant place and identity of the following vowel are not statistically independent, giving further problems for the idea that categorical phonetic or linguistic features have already been extracted at the stage where dichotic inputs are pooled. Until many more values of both the critical cues and the phonetic contexts are tried, the assignment of FSA and hence the material summation of dichotic features to a phonetic level of processing is premature.

\section{Summary}

This research explored primarily the notion that certain encoded acoustical properties in speech sounds may automatically exert a triggering effect resulting in their preferential processing by lefthemispheric speech analyzers. In demonstrating a REA in the absence of the feature thought most likely to exert such a triggering effect, namely, voice onset delay, we are led to conclude that the trigger feature is not a reasonable explanatory concept. However, the present results do provide direct support for the suggestion that the left hemisphere displays some specialization for processing formant transitions which cue phonemic distinctions. The data obtained also speak to the stages involved in the processing of phonetic features by showing that the so-called "feature sharing advantage" (FSA) is restricted to the feature shared, ruling out global response facilitation. The smaller FSA on dichotic pairs sharing voicing than on pairs sharing place was, together with other results, provisionally interpreted as evidence that the FSA may arise initially during auditory analysis or storage, rather than in a phonetic stage as is currently supposed.

\section{REFERENCE NOTES}

1. Haycock, K., \& Haggard, M. P. Pitch skip in stop consonants. Speech Synthesis and Perception Series 1, No. 3, 23-26. (Psychological Laboratory, University of Cambridge).

\section{REFERENCES}

Ades, A. E. A bilateral component in speech perception? Journal of the Acoustical Society of America, 1974, 56, 610-616.

AlleN, J. Determinants of lateral asymmetry and backward masking in dichotic speech perception. DPhil thesis, University of Sussex, 1976.

Allen, J., \& HAGgARn, M. P. Backward masking and asymmetry of processing for vowels differing in acoustical similarity. Quarterly Journal of Experimental Psychology, in press.

Brondennt, D. E. Decision and stress. London: Academic Press, 1971.

Curring, J. E. Two left-hemisphere mechanisms in speech perception. Perception \& Psychophysics, 1974, 16, 601-612.

Cutring, J. E. Auditory and linguistic processes in speech perception: Inferences from six fusions in dichotic listening. Psychological Review, 1976, 83. 114-140.

DARWIN, C. J. Ear differences in the recall of fricatives and vowels. Quarterly Journal of Experimental Psychology, 1971, 23, 46-62. DARWIN, C. J., \& BADDELEY, A. D. Acoustic memory and the perception of speech. Cognitive Psychology, 1974, 6, 41-60.

HaGgaRd, M. P. Selectivity in speech perception models. Zeitschrift für Phonetik. Sprachwissenschaft und Kommunikations-forschung. 1968. Band 21, Heft $1 / 2$.

HAGGARD, M. P. Encoding and the REA for speech signals. Quarterly Journal of Experimental Psychology, 1971. 23. 34-45. Haggard, M. P. Dichotic listening. In H. Leibowitz, H.-L. 
Teuber, \& R. Held (Eds.), Handbook of sensory physiology (Vol. VIII). New York: Springer Verlag, 1977.

Haggard, M. P., Ambler, S., \& Callow, M. Pitch as a voicing cue. Journal of the Acoustical Society of America, 1970, 47, 613-617.

Haggard, M. P., \& Parkinson, A. Stimulus and task factors as determinants of ear advantages. Quarterly Journal of Experimental Psychology, 1971, 23, 168-177.

KinsBoURNE, M. The control of attention by interaction between the hemispheres. In S. Kornblum (Ed.), Attention and performance, IV. New York: Academic Press, 1973.

Liberman, A. M., Delattre, P. C., \& Cooper, F. S. Some cues for the distinction between voiced and voiceless stops in initial position. Language and Speech, 1958, 1, 153-167.

Liberman, A. M., Cooper, F. S., Shankweiller, D., \& Studdert-Kennedy, M. Perception of the speech code. Psychological Review, 1967, 74, 431-461.

LISKER, L. Is it VOT or a first formant transition detector? Journal of the Acoustical Society of America, 1974, 57, 1547-1551.

Lisker, L., \& Abramson, A. S. A cross-language study of voicing in initial stops: Acoustical measurements. Word, 1964, 20, 324-422.

LUCE, R. D. Individual choice behaviour. New York: Wiley, 1959.

MAssaro, D. W. Preperceptual images, processing time and perceptual units in auditory perception. Psychological Review, 1972, 79. 124-145.

Myers, T. F. Asymmetry and attention in phonic decoding. Acta Psychologica, 1970, 33, 158-177.

Paur, D., \& Haggard, M. P. Parameter-specific voice-difference effects in dichotic CV identification. Journal of the Acoustical Society of America, 1975, 58, S75.
Pisoni, D. B. Dichotic listening and processing phonetic features. In F. Restle, R. M. Shiffrin, H. Castellan, H. Lindman, \& D. B. Pisoni (Eds.), Cognitive theory (Vol. 1). Hillsdale, N.J: Erlbaum, 1975.

Pisoni, D. B., \& MCNABB, S. D. Dichotic interactions of speech sounds and phonetic feature processing. Brain and Language, $1974,1,351-362$.

Porter, R. J., \& Mrrabile, P. J. Dichotic and monotic interaction between speech and nonspeech at different stimulus-onset asynchronies. Perception \& Psychophysics, in press.

Spellacy, F., \& Blumstein, S. The influence of language set on ear-preference in phoneme recognition. Cortex, 1970, 6, 430-439.

StEVENS, K. N., \& KLATT, D. H. Role of formant transitions in the voiceless distinction for stops. Joumal of the Acoustical Society of America, 1974, 55, 653-659.

StUdDert-Kennedy, M., \& Shankweiler, D. Hemispheric specialization for speech perception. Joumal of the Acoustical Society of America, 1970, 48, 579-594.

Studdert-Kennedy, M., Shankweiler, D., \& Pisoni, D. B. Auditory and phonetic processes in speech perception. Evidence from a dichotic study. Cognitive Psychology, 1972, 3, 455-466.

Summerfield, A. Q. First formant and frequency as a cue to stopconsonant voicing. Journal of the Acoustical Society of America, 1975, 58, S93.

Summerfield, A. Q., \& Haggard, M. P. Perceptual processing of multiple cues and contexts: Effects of following vowel on stop consonant voicing. Journal of Phonetics, 1974, 2, 279-295.

(Received for publication April 27, 1976; revision accepted October 4, 1976.) 